\section{Commentary: Size seems to matter, but it is not clear why}

\author{
Kathryn E. Engelhardt, MD, MS, and \\ Bryan F. Meyers, MD, MPH
}

Preoperative pulmonary artery hypertension (PA-HTN) can result in poor outcomes after lobectomy but requires invasive testing to formally diagnose. Enlargement of branch PAs may suggest PA-HTN. Because all patients undergoing pulmonary resection will have imaging allowing for PA measurement, surgeons may have access to a method of estimating which patients have subclinical PA-HTN and therefore higher risk of poor outcomes.

Kneuertz and colleagues ${ }^{1}$ report an association between PA size and lobectomy outcomes. Specifically, an increase in absolute PA size by $1 \mathrm{~mm}$ increased the odds of major complication by $12 \%$, unexpected intensive care unit admission by $11 \%$, and 30 -day mortality by $25 \%$. They did not find any association between outcomes and PA size adjusted for the patient's aortic diameter or vertebra size. In a subset with appropriate data, they compared PA size with right ventricular systolic pressure measured by echocardiography and did not see an association. There was no direct PA pressure measurement from invasive testing in this retrospective cohort. These findings are curious because the hypothesis here is that PA size serves as a surrogate for PA pressure.

As an initial foray into morphomics in thoracic surgery, this work offers a look at an alternative to invasive testing to assess surgical risk based on PA pressures. We see opportunities for next steps to make this science operational in the clinical setting. First, the authors included PA size in their models as a continuous variable. This allows one to conclude

\footnotetext{
From the Division of Cardiothoracic Surgery, Department of Surgery, Washington University School of Medicine/Barnes-Jewish Hospital, St Louis, Mo.

Disclosures: The authors reported no conflicts of interest.

The Journal policy requires editors and reviewers to disclose conflicts of interest and to decline handling or reviewing manuscripts for which they may have a conflict of interest. The editors and reviewers of this article have no conflicts of interest.

Received for publication Feb 1, 2021; revisions received Feb 1, 2021; accepted for publication Feb 3, 2021; available ahead of print Feb 12, 2021.

Address for reprints: Bryan F. Meyers, MD, MPH, Division of Cardiothoracic Surgery, Department of Surgery, Washington University School of Medicine/ Barnes-Jewish Hospital, Campus Box 8234, 660 S Euclid Ave, St Louis, MO 63110-1093 (E-mail: meyersb@wustl.edu).

J Thorac Cardiovasc Surg 2022;163:1530-1

$0022-5223 / \$ 36.00$

Copyright (c) 2021 by The American Association for Thoracic Surgery

https://doi.org/10.1016/j.jtcvs.2021.02.013
}

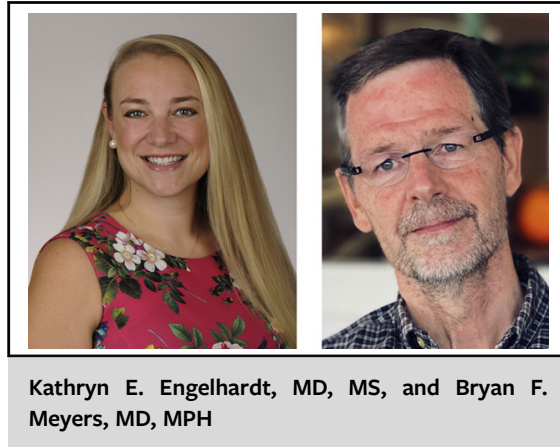

CENTRAL MESSAGE

Pulmonary artery size may be associated with lobectomy outcomes, but research is needed to understand this relationship and allow application to clinical practice.

that for $\mathrm{X}$ increase in size, there is a $\mathrm{Y}$ increase in risk of poor outcome. Ideally, we might envision a PA size cutoff above which patients are at sufficient risk to warrant at least further invasive testing and, at most, avoid surgery altogether. Inflection point analysis, restricted cubic splines analysis, or $\mathrm{J}$ point analysis may help determine cutoff values of PA size to guide clinical decision making. Second, as mentioned above, the authors reported an association between absolute size of the PA and outcomes, but when they adjusted PA size for the size of the patient, there was no association. Additionally, when they compared PA size with right ventricular systolic pressure as measured by transthoracic echocardiogram (shown in their Table E1), they found no association, thereby deflating the hypothesis that PA size acts as a surrogate for PA pressure. These observations are troubling and, based on the information presented in their discussion, the inconsistency bothers the authors, too. Further work should delineate whether it is PA pressure that is exerting a role in worse outcomes or if there is something else linked to both PA size and outcomes that is the driver or confounder here.

The authors have shown an association between larger PA size and worse survival, higher complication rate, and increased unexpected use of the intensive care unit. Future 
work should aim to understand the pathophysiology of this association, confirm the authors' findings in additional datasets, and delineate cutoff values useful to guide clinical care such as invasive testing.

\section{Reference}

1. Kneuertz PJ, Yudovich MS, Amadi CC, Bashian E, D'Souza DM, Abdel Rasoul M, et al. Pulmonary artery size on computed-tomography is associated with major morbidity after pulmonary lobectomy. J Thorac Cardiovasc Surg. 2022;163:1521-9.e2.
See Article page 1521.

\section{Commentary: Comparing apples to oranges can bear fruit}

\author{
Jonathan C. Nesbitt, MD
}

Pulmonary hypertension $(\mathrm{PH})$ is a recognized risk factor for perioperative morbidity and mortality. Select patients with PH can safely undergo pulmonary resection, but they remain at increased risk for postoperative complications. ${ }^{1}$

For patients being evaluated for pulmonary resection, considerations of risk determination include lung function (mechanics and physiology), exercise tolerance, and cardiac status (inherent disease and performance). The presence of $\mathrm{PH}$ is not commonly investigated and can occur in the absence of advanced pulmonary dysfunction or hypoxemia. The diagnosis of $\mathrm{PH}$ requires a high degree of suspicion and is established by hemodynamic measurements, most accurately during right-sided heart catheterization. Echocardiography is frequently used as a less invasive test, but it is limited in scope and accuracy.

Chest computed tomography (CT) is accepted as a fundamental study during preoperative assessment of patients undergoing pulmonary resection to assess the primary lesion and extent of disease. In radiologic interpretations of chest $\mathrm{CT}$ scans, the size of the pulmonary artery (PA) or comparative PA-to-aorta size (PA:Ao ratio) is not routinely reported. Yet the PA:Ao ratio or the PA size itself has been recognized as a surrogate indicator of $\mathrm{PH}$. In addition, the

\footnotetext{
From the Vanderbilt Thoracic Surgery, Vanderbilt University Medical Center, Nashville, Tenn

Disclosures: The author reported no conflicts of interest.

The Journal policy requires editors and reviewers to disclose conflicts of interest and to decline handling or reviewing manuscripts for which they may have a conflict of interest. The editors and reviewers of this article have no conflicts of interest.

Received for publication Feb 10, 2021; revisions received Feb 10, 2021; accepted for publication Feb 11, 2021; available ahead of print Feb 19, 2021.

Address for reprints: Jonathan C Nesbitt, MD, Thoracic Surgery, Vanderbilt University Medical Center, Suite 609, Oxford House, 1313 21st Ave South, Nashville, TN 37232 (E-mail: jon.nesbitt@vumc.org).

J Thorac Cardiovasc Surg 2022;163:1531-2

$0022-5223 / \$ 36.00$

Copyright (c) 2021 by The American Association for Thoracic Surgery

https://doi.org/10.1016/j.jtcvs.2021.02.042
}

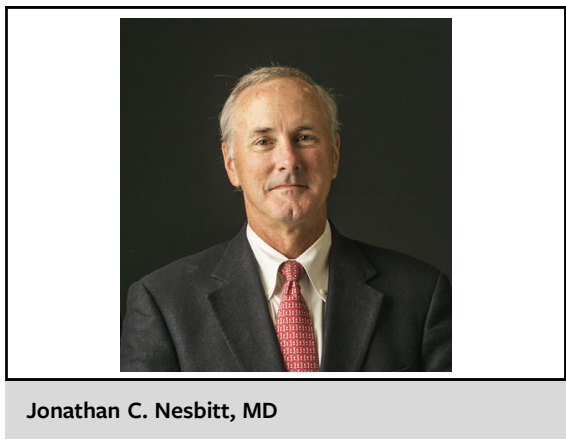

CENTRAL MESSAGE

Pulmonary artery enlargement

based on preoperative computed

tomography assessment may be

associated with increased major

operative risk and should alert the

surgeon to possible underlying

pulmonary hypertension.

PA:Ao ratio has been shown to be an accurate predictor of PH in patients with idiopathic pulmonary fibrosis and chronic obstructive pulmonary disease. Asakura and colleagues $^{2}$ used the PA:Ao ratio to determine outcomes and identified a PA:Ao ratio $>1.0$ as an independent predictor of postoperative complications following pulmonary resection for lung cancer.

In this issue of the Journal, Kneuertz and colleagues ${ }^{3}$ discuss their findings that PA size on preoperative chest $\mathrm{CT}$ is associated with increased risk of complications after lobectomy. Their well-conducted study on a large population from a single institution showed that comparative enlargement of same-side surgery PA diameter (ssPAD) was associated with increased major morbidity after lobectomy, independent of other known cardiopulmonary risk 